\title{
Análise do potencial da utilização de energia solar em Cuiabá/MT
}

A capital de Mato Grosso, Cuiabá, assim como o restante do estado, apresenta condições climáticas intensas ao longo do ano. Cidade de clima quente e com céu predominantemente ensolarado durante todo o ano, Cuiabá possui grande potencial para a implantação de energia solar fotovoltaica, apesar de não estar localizada na área do Brasil que recebe maior radiação. Este estudo tem como objetivo analisar este potencial, tendo em vista a importância do aproveitamento de energias renováveis no cenário atual e do aumento da demanda por energia elétrica no Brasil. A análise foi feita por meio dos dados de irradiação provenientes do software SunData, tanto no plano horizontal, quanto no plano inclinado ideal para a geração de energia, e a partir deles foi calculado o fator de capacidade de duas placas solares para a irradiação incidente na área urbana de Cuiabá/MT. Além disso, foi realizada uma análise do potencial na escala local a partir de modelagem 3D gerada pelo software Autodesk Revit em conjunto com o plugin Insight Analysis.

Palavras-chave: Clima; Energia; Radiação solar.

\section{Analysis of the potential of solar energy use in Cuiabá/MT}

The capital of Mato Grosso, Cuiabá, as well as the rest of the state, has intense weather conditions throughout the year. City of warm weather and with predominantly sunny sky all year round, Cuiabá has a great potential for the implantation of solar photovoltaic energy, although it is not located in the area of Brazil that receives more radiation. This study aims to evaluate this potential, considering the increase in the demand for electric energy in Brazil and the importance of the use of renewable energy in the current scenario. The evaluation was done through the irradiation data from the SunData software, both in the horizontal plane and in the ideal inclined plane for the generation of energy, and from them were calculated the capacity factor of two solar panels for the incident radiation in the urban area of Cuiabá/MT. In addition, a local scale potential analysis was performed from 3D modeling generated by Autodesk Revit software jointly with the Insight Analysis plug-in.

Keywords: Climate; Energy; Solar radiation.

Topic: Sistemas de Energia Sustentável

Reviewed anonymously in the process of blind peer.
Received: 12/04/2019

Approved: $27 / 05 / 2019$
Natália Amorim Magalhães

Universidade Federal de Mato Grosso, Brasil

http://lattes.cnpq.br/5052820068447580

natalia.amagalhaes@gmail.com

Felipe Gustavo Capovilla dos Santos

Universidade Federal de Mato Grosso, Brasil

http://lattes.cnpq.br/8776582215442698

felipe.capovilla@gmail.com

Emília Garcez da Luz (D)

Universidade Federal de Mato Grosso, Brasil

http://lattes.cnpq.br/5960137943241493

http://orcid.org/0000-0003-2621-6128

emiliagluz@gmail.com
Marta Cristina de Jesus Albuquerque Nogueira

Universidade Federal de Mato Grosso, Brasil

http://lattes.cnpq.br/8280601583280522

mcjanp@gmail.com

José de Souza Nogueira (iD

Universidade Federal de Mato Grosso, Brasi

http://lattes.cnpq.br/5893185001802390

http://orcid.org/0000-0003-2504-7924

parananogueira@gmail.com

Flávia Maria de Moura Santos

Universidade Federal de Mato Grosso, Brasil

http://lattes.cnpq.br/4000857814123856

flavia_mms@hotmail.com
Referencing this:

MAGALHÃES, N. A.; SANTOS, F. G. C.; LUZ, E. G.; NOGUEIRA, M. C. J. A.; NOGUEIRA, J. S.; SANTOS, F. M. M.. Análise do potencial da utilização de energia solar em Cuiabá/MT. Revista Ibero Americana de Ciências Ambientais, v.10, n.3, p.114-123, 2019. DOI: http://doi.org/10.6008/CBPC2179-6858.2019.003.0011

DOI: 10.6008/CBPC2179-6858.2019.003.0011 


\section{INTRODUÇÃO}

Autoridades e cientistas do planeta se voltam todos os anos em discussões e colocam em voga o aquecimento global. Além desta, são muitas as pautas relacionadas ao clima, tais como a poluição atmosférica e a preocupação com os recursos naturais presentes no globo, tendo em vista que o processo de industrialização e o desenvolvimento econômico vêm se estreitando cada vez mais e consumindo as fontes presentes no planeta de forma indiscriminada.

Hoje o sistema elétrico brasileiro está quase totalmente conectado ao Sistema Interligado Nacional (SIN), que, por meio da malha de transmissão, possibilita o suprimento de energia elétrica nas regiões interligadas eletricamente, permitindo assim, a obtenção de ganhos sinérgicos e explorando a diversidade entre os regimes hidrológicos das bacias (ANEEL, 2014; ONS, 2019).

De acordo com Pereira et al. (2017) aponta um crescimento da demanda de eletricidade na ordem de $200 \%$ para os próximos 30 anos, com base na série histórica do consumo de eletricidade no Brasil. Avaliando-se as características de suscetibilidade dos recursos hídricos em conjunto com o crescimento da demanda de eletricidade, nota-se a necessidade de se diversificar as fontes de energia que compõem a matriz energética brasileira, buscando aproveitar fontes de energia que gerem baixo impacto ao meio ambiente.

Além disso, com as questões ambientais cada vez mais em evidência no cenário mundial, viu-se a necessidade de uma fonte que, diferentemente dos combustíveis fosseis, considerados os responsáveis pelas mudanças climáticas no mundo, gere poucos impactos durante a sua produção e consumo. Estudos visando o desenvolvimento e avaliação de novas estratégias passivas capazes de garantir a sustentabilidade da construção aliada ao conforto do usuário são de extrema relevância. No Brasil, o uso da placa solar vem sendo muito difundido e incentivado, o que chama a atenção para estudos que comprovem a eficiência desta em detrimento da especulação do mercado, que visa apenas o lucro de sua implantação.

A geração de energia dentro dos limites da cidade pode, ainda, trazer muitas vantagens, como, por exemplo, o aumento da eficiência devido à redução das perdas de transmissão de energia (SARRALDE et al., 2015). O potencial de geração de energia pode ser otimizado a partir do uso da tecnologia associado ao aproveitamento da morfologia urbana, nas coberturas e nas fachadas das edificações. As edificações são um dos mais relevantes objetos do ponto de vista de produção de energia solar, e devem ser considerados como objetos de projeção de sombras e como coletores solares, cuja complexidade geométrica influencia no potencial fotovoltaico (REDWEIK et al., 2013).

Segundo Lopes (2013), a existência de fontes de sombreamento, como árvores e edifícios, pode provocar uma redução na produção de energia e, com isso, elevar o custo financeiro e retardar o retorno do investimento da unidade de microgeração. Diversos trabalhos já avaliaram o potencial da energia solar na malha urbana sob diferentes óticas, desde indicadores morfológicos (MOHAJERI et al., 2016; MORGANTI et al., 2017) à verificação do potencial pela integração com modelos 3D (KANTERS et al., 2012; MACHETE et al., 2018; PILI et al., 2018). A análise da energia solar na escala urbana pode ser realizada por ferramentas especializadas, como, por exemplo, os softwares Radiance, DIVA, CitySim, SUNtool (MORGANTI et al., 2017), 
ou mesmo extensões que podem ser utilizadas em conjunto a outros softwares, como as extensões Solar Energy Analysis, disponível para o SketchUp, e Insight Solar Analysis, disponível para o Revit, da Autodesk. Dessa maneira, este estudo busca avaliar o potencial solar da cobertura de edificações de uma área urbana delimitada na região central do município de Cuiabá com uma aproximação por modelagem 3D, a fim de aproveitar a radiação incidente elevada recebida pela região.

\section{METODOLOGIA}

\section{Área de estudo}

A cidade de Cuiabá, no estado de Mato Grosso, situada nas coordenadas 15o 35' 46" S e 56을' $45^{\prime \prime}$ W), possui o clima quente e dias majoritariamente ensolarados, com insolação de $5,364 \mathrm{kWh} \cdot \mathrm{m}^{-2}$.dia-1 no plano horizontal (SWERA, 2019).

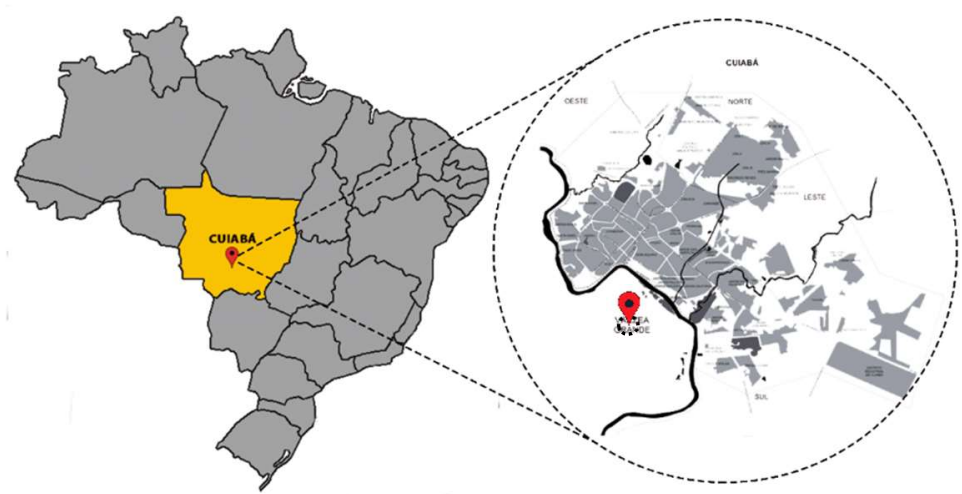

Figura 1: Mapa área de estudo.

Neste trabalho, a concepção do modelo 3D se deu a partir do projeto bidimensional da malha urbana do município de Cuiabá/MT, disponibilizado pela prefeitura municipal. A área selecionada (Figura 2) corresponde à região central da cidade, onde há uma conglomeração de prédios públicos, em um raio de 200m, que coincide com o raio mínimo que define uma zona climática local (STEWART et al., 2012).

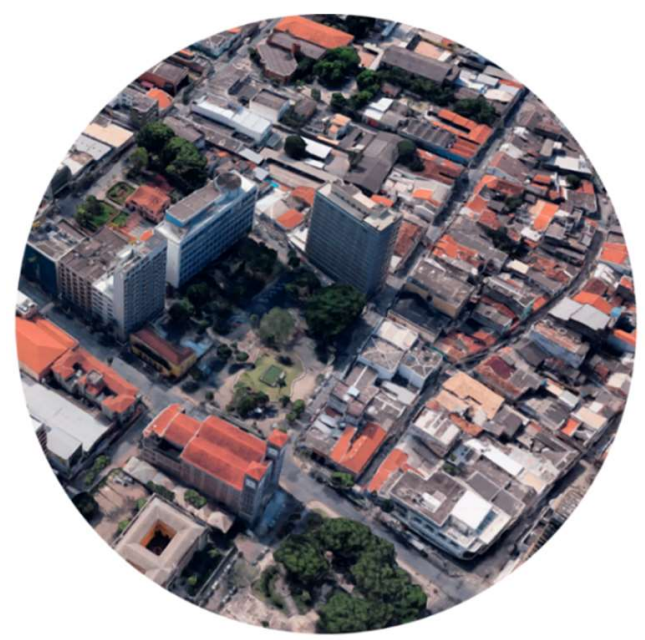

Figura 2: Perspectiva Real.

A área de estudo se encontra na região centro-norte de Cuiabá/MT. Área caracterizada por predominância de edifícios de caráter comercial e institucional. Levando em consideração os usos das 
edificações, vale ressaltar que estas demandam maior uso de energia elétrica devido à necessidade de climatização por ar condicionado durante todo o período comercial do dia.

O gabarito (alturas das edificações) no local é bastante variado, sendo os próprios edifícios existentes no local os agentes ativos de sombreamento em áreas adjacentes. Este foi um ponto importante para a escolha da área de estudo, tendo em vista que o sombreamento é um fator muito importante na interferência no potencial de placas solares, tendo em vista a redução da radiação que chega diretamente nestas. Com a região de estudo delimitada, foi iniciado o processo de elevação dos volumes da malha urbana no software para BIM (Building Information Modelling - Modelagem de Informações de Construção) da Autodesk, Revit, na sua versão estudante, fornecida para fins acadêmicos sem custos.

\section{Análise de insolação na escala local}

Para que a placa solar possua um bom potencial de geração de energia existem algumas variáveis a serem analisadas e seguidas, tais como a avaliação de sua eficiência e potência, inclinação ideal para determinada região, entre outros. Sendo assim, este estudo servirá para analisar se Cuiabá realmente possui o potencial adequado para a implantação de energia solar.

A posição do painel deve ser determinada pela latitude de seu local de instalação e orientação de maior incidência de insolação, o que, no hemisfério Sul, corresponde ao Norte geográfico. Deve, também, ser considerada a altura solar, que especifica a localização do Sol no espaço. O Sol se nasce e se põe em diferentes localizações na Terra, e descreve trajetórias diferentes em cada dia do ano: a altura do Sol, por exemplo, é maior nos dias de verão, enquanto no inverno a trajetória descrita é mais baixa (VILLALVA et al., 2012).

O melhor aproveitamento da radiação solar direta ocorre quando os raios incidem perpendicularmente ao painel fotovoltaico. Sendo assim, o ideal seria que o painel acompanhasse o movimento do Sol, permitindo a maior geração possível durante o dia. Porém, os painéis são instalados em uma posição fixa e, portanto, o ângulo de inclinação do painel a ser determinado deve ser aquele que permita uma maior exposição do painel aos raios solares incidentes.

Cronemberger et al. (2012) avaliaram 78 cidades brasileiras e verificaram que o ângulo ótimo de inclinação está compreendido entre a latitude e o valor excedido em até $9^{\circ}$. Sendo assim, Cuiabá, por se encontrar no Hemisfério Sul e estar localizada entre as latitudes de $10^{\circ}$ e $20^{\circ}$, possui um ângulo ótimo de inclinação da placa solar de $16^{\circ}$, correspondente exatamente à sua latitude.

De acordo com Machete et al. (2018), para analisar mais precisamente a insolação que chega até às coberturas dos edifícios no meio urbano é necessário que se faça a avaliação por um modelo 3D do contexto urbano, que esteja apto a responder a diferentes escalas espaciais de análise, que pode ser aplicado desde uma determinada edificação até uma área urbana de interesse.

A elevação foi feita em conjunto com a análise do mapa topográfico e dos dados geoespaciais, disponíveis em imagens de satélites e fotografias ao nível da rua. Por meio da ferramenta de extrusão do polígono, a representação volumétrica foi concebida com base nos atributos das edificações, como número 
de andares e de ambientes. A modelagem da forma 3D dos edifícios permite a avaliação da exposição solar, assim como das sombras projetadas pelos sólidos (MACHETE et al., 2018).

A análise da exposição solar foi feita utilizando o plugin Insight Solar Analysis, distribuído pela Autodesk, em conjunto com o Revit. O Insight Solar Analysis permite avaliar o desempenho e visualizar os impactos da radiação solar nas faces dos sólidos, pela quantificação da distribuição e intensidade da radiação incidente, considerando os efeitos de sombreamento de objetos adjacentes, como vegetação e edifícios no entorno.

\section{Análise do potencial fotovoltaico}

Para a análise do potencial do uso de energia solar, primeiramente foi utilizado o programa SunData - elaborado pelo CRESESB - Centro de Referência Para Energia Solar, para a obtenção dos dados de irradiação solar ( $\mathrm{kWh} / \mathrm{m} 2$.dia). A irradiação é o melhor formato para se dimensionar a quantidade de energia que um conjunto fotovoltaico pode gerar. Para o dimensionamento da quantidade de energia que um conjunto fotovoltaico pode gerar, foram escolhidos dois módulos fotovoltaicos da Tabela de Eficiência Energética do Programa Brasileiro de Etiquetagem do INMETRO, todos de classificação A (mais eficiente). As características relevantes dos módulos escolhidos estão descritas na Tabela 1.

Tabela 1: Características dos módulos fotovoltaicos escolhidos.

\begin{tabular}{|l|l|l|l|}
\hline MODULO 1 & MODULO 2 \\
\hline Material & Silício Policristalino & Material & Silício Policristalino \\
\hline Área & $1,61 \mathrm{~m}^{2}$ & Área & $1,61 \mathrm{~m}^{2}$ \\
\hline Potência & $235 \mathrm{~W}$ & Potência & $250 \mathrm{~W}$ \\
\hline Eficiência & $14,6 \%$ & Eficiência & $15,5 \%$ \\
\hline
\end{tabular}

Ambos painéis escolhidos para análise são da marca Canadian Solar (Figura 3) e de silício policristalino, tendo em vista que este amplamente utilizado no mercado. Além disso, a quantidade de silício residual gerado durante o processo de corte das células fotovoltaicas é menor em comparação com monocristalino e tem um custo financeiro menor. A vida útil dos painéis policristalinos é maior que 30 anos sua garantia é de 25 anos.

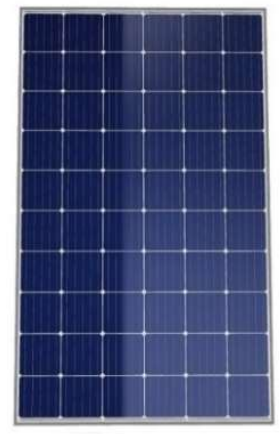

Figura 3: Placa solar fotovoltaica.

Com as características dos módulos fotovoltaicos utilizados, é possível se dimensionar a energia gerada (E) com a irradiação obtida de acordo com os dados do software SunData, através da Equação 1.

$$
\mathrm{E}=\eta \cdot \mathrm{A} \cdot \mathrm{H}_{\text {glob,painel }}
$$


$\eta$ - eficiência do módulo fotovoltaico, em valor percentual (0,12 para 12\%, por ex.); A - área ocupada pelo módulo, em $\mathrm{m}^{2} ; \mathrm{e}$

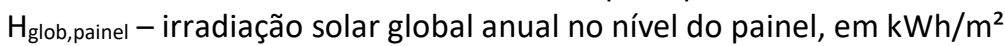

Ao utilizar essa equação para o cálculo da energia, assume-se que não houve nenhum tipo de perda durante a geração. Onde $\eta$ é a eficiência do modulo fotovoltaico, A é a área ocupada por este e HT a irradiação solar incidente sobre ele. $O$ fator de capacidade é, então, calculado de acordo com a Equação 2.

$$
\mathrm{FC}=\frac{\mathrm{H}_{\text {glob.painel }}}{\mathrm{P} \times \mathrm{t}}
$$

Onde: E - energia consumida ou gerada em determinado período de tempo, em MWh; $\mathrm{P}$ - potência instalada da geração, em MW; e $\mathrm{t}$ - tempo do período de medição da energia, em $\mathrm{h}$

$\mathrm{Na}$ equação do fator de capacidade divide-se o que foi obtido anteriormente de energia gerada em MW pela potência multiplicada por 24 horas do dia, pelos dias do ano (365). Sendo assim, são calculados fatores de capacidade para cada mês do ano utilizando as suas características médias, e a análise foi feita a partir do fator de capacidade médio anual.

\section{RESULTADOS}

\section{Análise de insolação na escala local}

Segundo o Atlas Brasileiro de Energia Solar (PEREIRA et al., 2017) a demanda crescente de energia elétrica, representada pelo uso crescente de aparelhos de ar-condicionado, vem alterando o perfil de consumo e demanda no Brasil. Ano a ano os valores máximos de demanda são registrados nos meses de verão e no horário entre 12 e 15 horas, com excelente coincidência com a disponibilidade de radiação solar para a geração fotovoltaica.

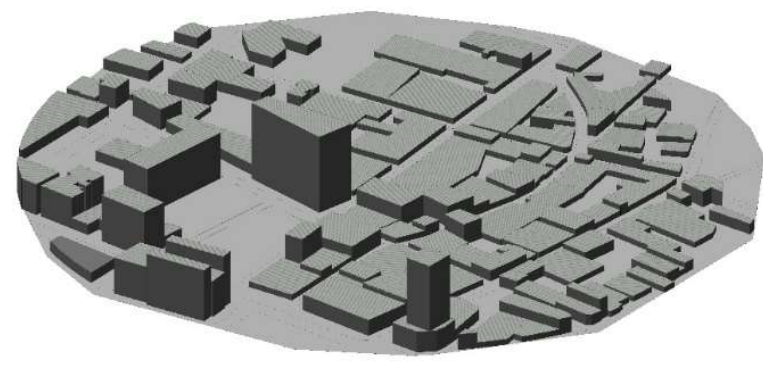

Figura 4: Perspectiva para Estudo.

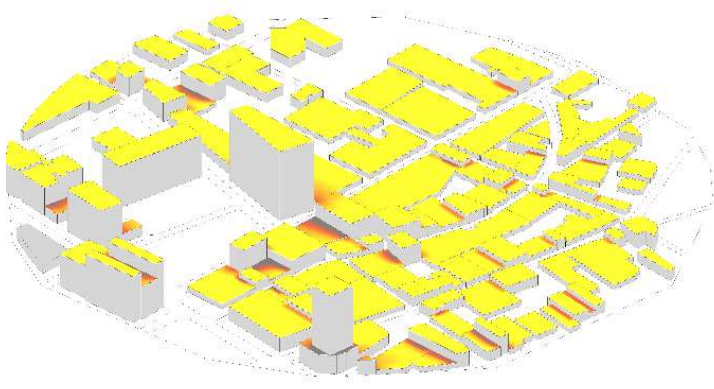

Figura 5: Análise do Potencial Fotovoltaico.

De acordo com os dados obtidos com o plugin Insight Analysis, a maior parte da área de estudo apresenta níveis de irradiação na ordem de 1.500 a $1.655 \mathrm{kWh} \cdot \mathrm{m}^{-2}$.ano-1 , que é um potencial satisfatório quando se considera que a região do Brasil com maior incidência de irradiação recebe cerca de 2070 kWh.m${ }^{2}$.ano ${ }^{-1}$ (PEREIRA et al., 2017).

Com raio de 200 metros e uma área total de $125.600 \mathrm{~m}^{2}$, seguindo a estimativa obtida de coberturas e potencial fotovoltaico, foram encontrados cerca de $62.000 \mathrm{~m}^{2}$ de coberturas de edificações, resultando uma irradiação recebida de aproximadamente $100.000 .000 \mathrm{kWh}$ na região estudada anualmente. Além disso, 
vale frisar que, tais dados podem ser validados devido ao georreferenciamento preciso entre a posição geográfica e o caminhamento do sol na localidade realizado pelo software Autodesk Revit.
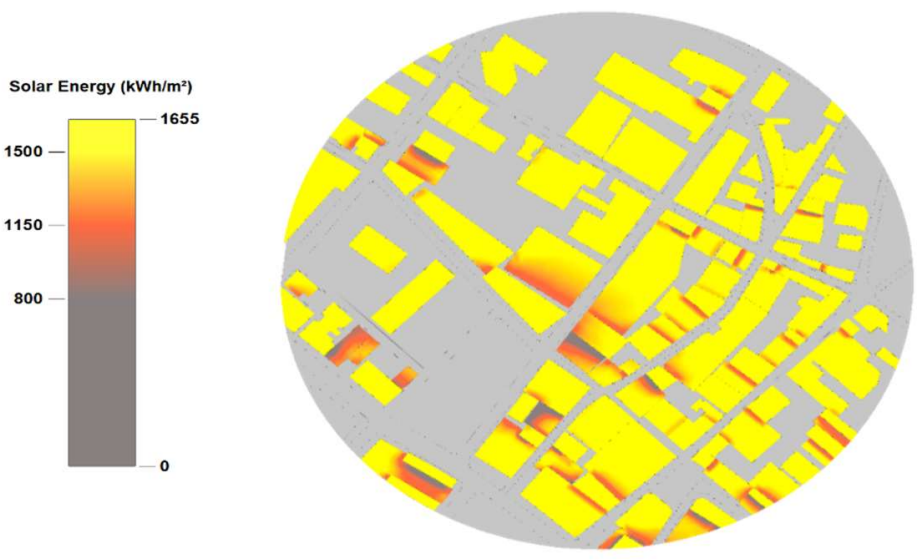

Figura 6: Coberturas e Potenciais Fotovoltaicos.

\section{Análise do potencial fotovoltaico}

O primeiro resultado foi obtido a partir do programa SunData. Nele foram encontrados os valores de irradiação média diária no plano horizontal e no plano inclinado a 16으, conforme mostra a Tabela 2. Apesar da irradiação solar diária média calculada a partir dos dados meteorológicos e dos valores obtidos através do programa SunData apresentarem um valor satisfatório, já que usa como referência os dados do Atlas Brasileiro de Energia Solar (PEREIRA et al., 2017), esses valores não são suficientes para mostrar a viabilidade da utilização das placas solares na cidade de Cuiabá/MT. Foram calculados, então, fatores de capacidade para os dois módulos de placas solares caracterizados anteriormente. A partir dos dados de irradiação obteve-se os valores de energia gerada e do fator de capacidade médio anual para a cidade de Cuiabá/MT, nos planos horizontal (Tabela 3) e inclinado a $16^{\circ}$ (Tabela 4).

Tabela 2: Irradiação média diária e mensal nos planos horizontal e inclinado para Cuiabá/MT.

\begin{tabular}{|c|c|c|c|c|}
\hline \multirow{3}{*}{ Mês } & \multicolumn{2}{|c|}{ Plano Horizontal } & \multicolumn{2}{|c|}{ Plano Inclinado 16음 } \\
\hline & \multicolumn{2}{|l|}{ Irradiação } & \multicolumn{2}{|l|}{ Irradiação } \\
\hline & (kWh/m².dia) & (kWh/m².mês) & & (kWh/m².dia) \\
\hline Janeiro & 5,44 & 168,64 & Janeiro & 5,44 \\
\hline Fevereiro & 5,29 & 148,12 & Fevereiro & 5,29 \\
\hline Março & 5,14 & 159,34 & Março & 5,14 \\
\hline Abril & 4,9 & 147 & Abril & 4,9 \\
\hline Maio & 4,45 & 137,95 & Maio & 4,45 \\
\hline Junho & 4,42 & 132,6 & Junho & 4,42 \\
\hline Julho & 4,57 & 141,67 & Julho & 4,57 \\
\hline Agosto & 5,43 & 168,33 & Agosto & 5,43 \\
\hline Setembro & 5,1 & 153 & Setembro & 5,1 \\
\hline Outubro & 5,28 & 163,68 & Outubro & 5,28 \\
\hline Novembro & 5,53 & 165,9 & Novembro & 5,53 \\
\hline Dezembro & 5,65 & 175,15 & Dezembro & 5,65 \\
\hline
\end{tabular}

Fonte: Software SunData, CRESESB (2019).

Tabela 3: Fator de capacidade no Plano Horizontal.

\begin{tabular}{|l|l|l|l|}
\hline Módulo 1 & Módulo 2 \\
\hline Energia gerada (MW) & Fator de capacidade & Energia gerada (MW) & Fator de capacidade \\
\hline 0,35 & $16,9 \%$ & 0,37 & $16,9 \%$ \\
\hline 0,37 & $18,2 \%$ & 0,40 & $18,2 \%$ \\
\hline
\end{tabular}




\begin{tabular}{|l|l|l|l|}
\hline 0,35 & $16,8 \%$ & 0,37 & $16,8 \%$ \\
\hline 0,32 & $15,8 \%$ & 0,34 & $15,7 \%$ \\
\hline 0,31 & $15,1 \%$ & 0,33 & $15,1 \%$ \\
\hline 0,33 & $16,2 \%$ & 0,35 & $16,1 \%$ \\
\hline 0,40 & $19,2 \%$ & 0,42 & $19,2 \%$ \\
\hline 0,36 & $17,5 \%$ & 0,38 & $17,4 \%$ \\
\hline 0,38 & $18,7 \%$ & 0,41 & $18,7 \%$ \\
\hline 0,39 & $18,9 \%$ & 0,41 & $18,9 \%$ \\
\hline 0,41 & $20,0 \%$ & 0,44 & $20,0 \%$ \\
\hline FC Médio & $17,71 \%$ & FC Médio & $17,68 \%$ \\
\hline
\end{tabular}

Fonte: Software SunData, CRESESB (2019).

Tabela 4: Fator de capacidade no Plano Inclinado a $16^{\circ}$.

\begin{tabular}{|l|l|l|l|}
\hline Módulo 1 & Mator de capacidade & Energia gerada (MW) & Fator de capacidade \\
\hline Energia gerada (MW) & $17,8 \%$ & 0,39 & $17,8 \%$ \\
\hline 0,37 & $16,3 \%$ & 0,36 & $16,3 \%$ \\
\hline 0,34 & $18,4 \%$ & 0,40 & $18,4 \%$ \\
\hline 0,38 & $18,1 \%$ & 0,40 & $18,0 \%$ \\
\hline 0,37 & $17,8 \%$ & 0,39 & $17,8 \%$ \\
\hline 0,37 & $17,7 \%$ & 0,39 & $17,6 \%$ \\
\hline 0,36 & $18,7 \%$ & 0,41 & $18,7 \%$ \\
\hline 0,38 & $21,3 \%$ & 0,46 & $21,2 \%$ \\
\hline 0,44 & $18,2 \%$ & 0,40 & $18,2 \%$ \\
\hline 0,37 & $18,3 \%$ & 0,40 & $18,3 \%$ \\
\hline 0,38 & $17,8 \%$ & 0,39 & $17,8 \%$ \\
\hline 0,37 & $18,4 \%$ & 0,40 & $18,4 \%$ \\
\hline 0,38 & $18,24 \%$ & FC Médio & $18,20 \%$ \\
\hline FC Médio & & \\
\hline
\end{tabular}

Fonte: Software SunData, CRESESB (2019).

Observa-se que, mesmo em casos de irradiações solares distintas, os fatores de capacidade médios apresentam valores muito parecidos, porém vale frisar que o fator de capacidade calculado para a situação onde a placa estaria inclinada em 16o, maior que os demais. A diferença entre os fatores de capacidade calculados para as duas situações é apresentada na Figura 7.

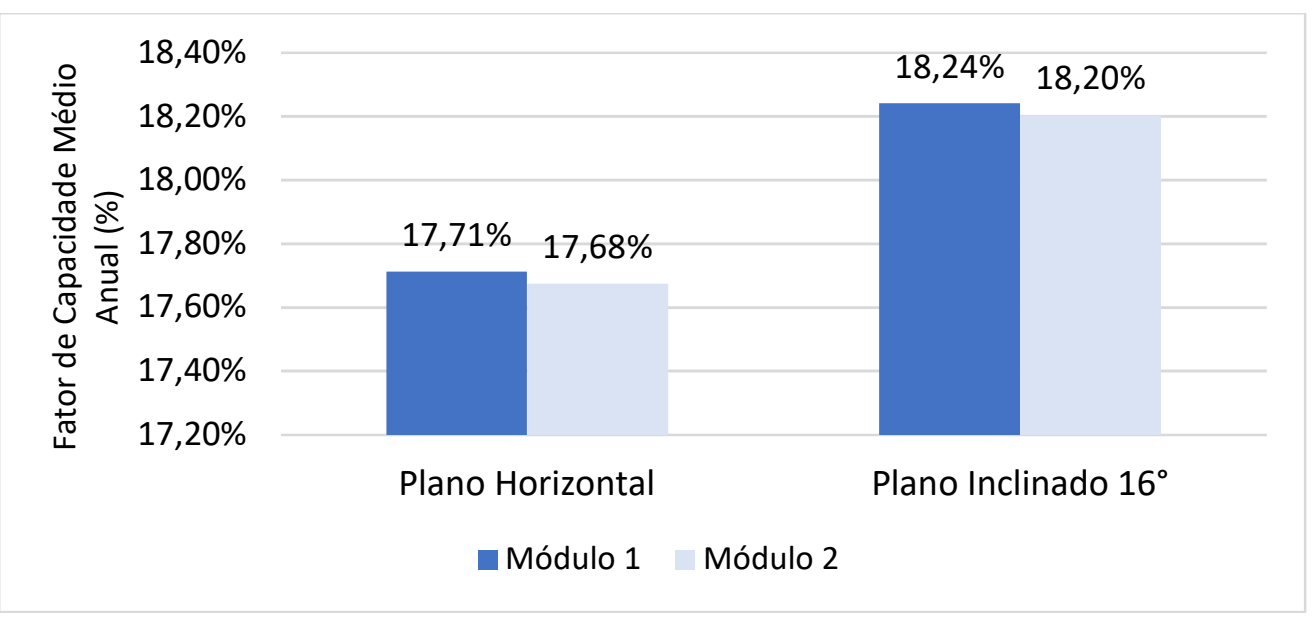

Figura 7: Gráfico comparativo Fatores de Capacidade Médios Anuais.

\section{DISCUSSÃO}

Em uma nota técnica lançada pela EPE (2012) Análise da Inserção da Geração Solar na Matriz Elétrica Brasileira, foram realizadas simulações quanto a viabilidade e a competitividade da geração solar na geração distribuída (isto é, de pequeno porte, em residências ou comércios). Considerando um painel solar com Fator de Capacidade de 15,1\%, concluiu-se que a sua utilização apresenta competitividade econômica quando 
comparada à energia fornecida pela concessionária local considerando uma vida útil do painel fotovoltaico de 20 anos, salvo em situações onde a tarifa de energia elétrica da concessionária local apresenta valores elevados.

A nota técnica afirma, ainda, que aplicações com fatores de capacidade maiores que os $15,1 \%$ se apresentam ainda mais competitivas. Como o menor valor observado de acordo com as placas adotadas foi exatamente de $15,1 \%$, pode-se concluir que a utilização dos painéis solares fotovoltaicos na produção de energia elétrica se mostra viável para a irradiação solar medida na cidade de Cuiabá/MT.

\section{CONCLUSÕES}

Com o aumento do consumo de energia elétrica no Brasil e no mundo e o consequente aumento dos impactos ambientais associados às suas fontes de produção que atualmente compõe a matriz energética mundial, há uma necessidade evidente de se ampliar a utilização de fontes consideradas limpas (ou renováveis) para a produção de energia elétrica.

A matriz energética brasileira é composta, majoritariamente, por energia advinda de fontes renováveis, sendo a principal a energia hidroelétrica. As condições hidrológicas desfavoráveis pelas quais o país passa atualmente, porém, fizeram com que a participação da energia hidroelétrica na matriz energética diminuísse nos últimos anos. Buscando evitar a utilização em maior escala de energias oriundas de fontes não-renováveis (como as termoelétricas), é necessário se pensar em fontes alternativas renováveis para a produção de energia, como a solar e a eólica.

Este estudo identificou que existe potencial satisfatório quanto a utilização da energia solar dentro do tecido urbano da cidade de Cuiabá/MT para a produção de energia elétrica, partir de modelagem 3D na escala local em região importante da cidade. Além disso, reforçou-se tal potencial utilizando-se de dados advindos do programa SunData - CRESESB. A partir de estudos já elaborados por Pereira et al. (2017), era esperado, em linhas gerais, que a energia solar apresentasse grande potencial de utilização nesta região.

Porém, no Brasil e, principalmente, na região Centro-Oeste, ainda se necessitam de mais estudos e de uma ampla discussão envolvendo governos, concessionárias, universidades, centros de pesquisa, etc., na razão de definir uma estratégia a longo prazo para a energia solar fotovoltaica conectada à rede do país, tendo em vista que existe uma demanda energética crescente e uma disponibilidade de radiação solar em níveis ótimos para aproveitar esta tecnologia no estado e no país.

\section{REFERÊNCIAS}

ANEEL. Resolução Normativa n. 622: Dispõe sobre as garantias financeiras e a efetivação de registros de contratos de compra e venda de energia elétrica, associados à comercialização no âmbito da Câmara de Comercialização de Energia Elétrica e dá outras providências. 2014.

CRESESB. Centro de referência para energia solar e eólica. Potencial energético solar. SunData: Base de dados sobre radiação solar no Brasil. 2019.
CRONEMBERGER, J.; MARTÍN, E. C.; SÁNCHEZ, S. V. Assessing the solar irradiation potential for solar photovoltaic applications in buildings at low latitudes: Making the case for Brazil. Energy and Buildings, v.55, p.264-272, 2012.

EPE. Empresa de pesquisa energética. Análise da inserção da geração solar na matriz elétrica brasileira. Nota Técnica da EPE. Rio de Janeiro: EPE, 2012. 
KANTERS, J.; HORVAT, M.. Solar energy as a design parameter in urban planning. Energy Procedia, v.30, p.11431152, 2012.

LOPES, R. J. C.. Efeito do sombreamento nos painéis fotovoltaicos. Dissertação (Mestrado em Engenharia Eletrotécnica) - Instituto Superior de Engenharia de Lisboa, Lisboa, 2013.

MOHAJERI, N.; UPADHYAY, G.; GUDMUNDSSON, A.; ASSOULINE, D.; KÄMPF, J.; SCARTEZZINI, J. L.. Effects of urban compactness on solar energy potential. Renewable Energy, v.93, p.469-482, 2016.

MORGANTI, M.; SALVATI, A.; COCH, H.; CECERE, C.. Urban morphology indicators for solar energy analysis. Energy Procedia, v.134, p.807-814, 2017. DOI: http://doi.org/10.1016/i.egypro.2017.09.533

ONS. Operador Nacional do Sistema Elétrico. Sobre o SIN: O que é o SIN. 2019.

PEREIRA, B. E.; MARTINS, F. R.; ABREU, S. L.; RUTHER, R.. Atlas Brasileiro de Energia Solar. 2 ed. São José dos Campos: SWERA, 2017.
PILI, S.; DESOGUS, G.; MELIS, D.. A GIS tool for the calculation of solar irradiation on buildings at the urban scale, based on Italian standards. Energy and Buildings, p.629-646, 2018

REDWEIK, P.; CATITA, C.; BRITO, M.. Solar energy potential on roofs and facades in an urban landscape. Solar Energy, v.97, p.332-341, 2013.

SARRALDE, J. J.; QUINN, D. J.; WIESMANN, D.; STEEMERS, K. Solar energy and urban morphology: Scenarios for increasing the renewable energy potential of neighbourhoods in London. Renewable Energy, v.73, p.10-17, 2015.

STEWART, I. D.; OKE, T. R.. Local Climate Zones for Urban Temperature Studies. Bulletin of the American Meteorological Society, v.93, n.12, p.1879-1900, 2012.

SWERA. Solar and Wind Energy Resource Assessment Programme. UNEP, 2019

VILLALVA, M. G.; GAZOLI, J. R.. Energia Solar Fotovoltaica: Conceitos e Aplicações: Sistemas Isolados e Conectados à Rede. São Paulo: Érica, 2012.

A CBPC - Companhia Brasileira de Produção Científica (CNPJ: 11.221.422/0001-03) detém os direitos materiais desta publicação. Os direitos referem-se à publicação do trabalho em qualquer parte do mundo, incluindo os direitos às renovações, expansões e disseminações da contribuiç̃o, bem como outros direitos subsidiários. Todos os trabalhos publicados eletronicamente poderão posteriormente ser publicados em coletâneas impressas sob coordenação da Sustenere Publishing, da Companhia Brasileira de Produção Científica e seus parceiros autorizados. Os (as) autores (as) preservam os direitos autorais, mas não têm permissão para a publicação da contribuição em outro meio, impresso ou digital, em português ou em tradução. 